\title{
Maximal averages over
}

\section{flat radial hypersurfaces}

Alex Iosevich

Let $A_{t} f(x)=\int_{S} f(x-t y) d \sigma(y)$, where $S$ is a smooth compact hypersurface in $\mathbb{R}^{n}$ and $d \sigma$ denotes the Lebesgue measure on $S$. Let $\mathcal{A} f(x)=\sup _{t>0}\left|A_{t} f(x)\right|$. If the hypersurface $S$ has non-vanishing Gaussian curvature, then

$$
\|\mathcal{A} f\|_{L^{p}\left(\mathbb{R}^{n}\right)} \leq C_{p}\|f\|_{L^{p}\left(\mathbb{R}^{n}\right)}, \quad f \in \mathcal{S}\left(\mathbb{R}^{n}\right),
$$

for $p>n /(n-1)$. Moreover, the result is sharp. See [St76], [Gr82].

If the hypersurface $S$ is convex and the order of contact with every tangent line is finite, the optimal exponents for the inequality $(*)$ are known in $\mathbb{R}^{3}$, (see [IoSaSe98]), and in any dimension in the range $p>2$, (see [IoSa96]). More precisely, the result in the range $p>2$ is the following.

Theorem 1 ([IoSa96]). Let $S$ be a smooth convex compact finite type hypersurface, in the sense that the order of contact with every tangent line is finite. Then for $p>2$, the following condition is necessary and sufficient for the maximal inequality $(*)$

$$
(d(x, \mathcal{H}))^{-1} \in L^{1 / p}(S),
$$

for every tangent hyperplane $\mathcal{H}$ not passing through the origin, where $d(x, \mathcal{H})$ denotes the distance from a point $x \in S$ to the tangent hyperplane $\mathcal{H}$. 
In fact, the condition (1) is a necessary condition for any smooth compact hypersurface in $\mathbb{R}^{n}$. See [IoSa96, Theorem 2].

In this paper we shall consider convex radial hypersurfaces of the form

$$
S=\left\{x \in B: x_{n}=\gamma\left(\left|x^{\prime}\right|\right)+1\right\}
$$

where $B$ is a ball centered at the origin, $x=\left(x^{\prime}, x_{n}\right), \gamma$ is convex, $\gamma$, $\gamma^{\prime \prime}$ increasing, $\gamma(0)=\gamma^{\prime}(0)=0$, and $\gamma$ is allowed to vanish of infinite order.

If $\gamma^{\prime \prime}$ does vanish of infinite order, the condition (1) cannot hold for any $p<\infty$. Since the condition (1) is necessary by Theorem 1 above, our only hope is to look for an inequality of the form

$$
\|\mathcal{A} f\|_{L^{\Phi}\left(\mathbb{R}^{n}\right)} \leq C_{\Phi}\|f\|_{L^{\Phi}\left(\mathbb{R}^{n}\right)}
$$

where $L^{\Phi}\left(\mathbb{R}^{n}\right)$ is an Orlicz space, near $L^{\infty}\left(\mathbb{R}^{n}\right)$, associated to a Young function $\Phi$, with the norm given by

$$
\|f\|_{\Phi}=\inf \left\{s>0: \int \Phi\left(\frac{|f(x)|}{s}\right) d x \leq 1\right\} .
$$

The following result was proved in [Bak95].

Theorem 2. Let $S$ be as in (2) with $n=3$. Assume that for each $\lambda>1$

$$
\frac{\gamma^{\prime}(\lambda t)}{\gamma^{\prime}(t)} \quad \text { is non-decreasing for } t>0
$$

Put $G(t)=t^{2} \gamma^{\prime}(t)$. For $\beta>1$ and $d>0$ let $\phi:[0, \infty) \longrightarrow[0, \infty)$ be a non-decreasing function such that $\phi(t)=t^{-1}\left(G\left(t^{-d}\right)\right)^{-\beta}$ if $t$ is sufficiently large, $\phi(t)>0$ if $t>1$, and $\phi(t)=0$ if $0 \leq t \leq 1$. Let $\Phi(u)=\int_{0}^{u} \phi(t) d t$. Then for every $d>1 / 2$ there exists a constant $C$ such that the estimate (3) holds.

The examples show (see [Bak95, Example 3.3]) that Theorem 2 is sharp for some surfaces, for example if $\gamma(s)=e^{-1 / s^{b}}, b>0$, but not for others, for example if $\gamma(s)=s^{m}$.

In this paper we shall give a set of simple sufficient conditions for the inequality (3) for some classes of Orlicz functions $\Phi$. We will show 
that our result is sharp for a wide class of both finite type and infinite type $\gamma$ 's.

\section{Assumptions on $\Phi$.}

Assume that $\Phi$ is a Young function such that $\Phi(s)=\int_{0}^{s} \phi(t) d t$, where $\phi:[0, \infty) \longrightarrow[0, \infty)$ is a non-decreasing function such that $\phi(t)=0$ for $0 \leq t \leq 1$, and $\phi(t)>0$ for $t>1$. Assume that there exist constants $c>1, C_{0}$, and $C_{1}$ such that

$$
\int_{1}^{u} \frac{\phi(t)}{t^{r}} d t \leq C_{0} \frac{\phi(u)}{u^{r-1}}, \quad \text { for } u>1,
$$

and for every $\lambda>1$,

$$
C_{1} \frac{\phi(\lambda t)}{\phi(t)} \geq \phi(\lambda), \quad \text { for } t \geq c .
$$

Our main reason for making these assumptions about $\Phi$ is the following generalization of the Marcienkiewicz interpolation theorem due to Bak. See [Bak95, Lemma 1.1].

Lemma 3. Let $r \in[1, \infty)$. Suppose that the operator $T$ is simultaneously weak type $(1,1)$ and $(\infty, \infty)$, namely there exist constants $A, B>0$ such that

$$
\begin{gathered}
\mu(\{x:|T f(x)|>t\}) \leq\left(\frac{A\|f\|_{r}}{t}\right)^{r}, \quad \text { for all } t>0, \\
\|T f\|_{\infty} \leq B\|f\|_{\infty} .
\end{gathered}
$$

Suppose that $\Phi$ satisfies the assumptions above. Then there exists a constant $C=C(\Phi, r)$ depending only on $\Phi$ and $r$ such that

$$
\|T f\|_{\Phi} \leq C B \Phi^{-1}\left(\left(\frac{A}{B}\right)^{r}\right)\|f\|_{\Phi} .
$$

REMARK. Lemma 3 has the following interesting consequence. Let

$$
\mathcal{A} f(x)=\sup _{t>0} \int f\left(x-t\left(s, s^{m}+1\right)\right) \psi(s) d s, \quad m>2,
$$


where $\psi$ is a smooth cutoff function, and let $\mathcal{A}^{k} f(x)$ denote the same operator with $s$ localized to the interval $\left[2^{-k}, 2^{-k+1}\right]$. It was proved in [I94] that $\mathcal{A}^{k}: L^{p}\left(\mathbb{R}^{2}\right) \longrightarrow L^{p}\left(\mathbb{R}^{2}\right), p>2$, with norm $C 2^{-k} 2^{m k / p}$. Let $\Phi_{p, \alpha}(t)=t^{p} \log ^{\alpha}(t)$. It follows by Lemma 3 that $\mathcal{A}: L^{\Phi_{p, \alpha}}\left(\mathbb{R}^{2}\right) \longrightarrow$ $L^{\Phi_{p, \alpha}}\left(\mathbb{R}^{2}\right)$ if $p=m$ and $\alpha>m$.

\section{Statement of results.}

Our main results are the following.

Theorem 4. Let $S$ be as in (2). Let $n \geq 3$. Suppose that $\Phi$ satisfies the conditions (6) and (7) above. Suppose that $\lim _{t \rightarrow 0} \Phi(t) / t^{2}=0$. Then the estimate (3) holds if

$$
\sum_{j=0}^{\infty} 2^{-j(n-1)} \Phi^{-1}\left(\frac{1}{\gamma\left(2^{-j}\right)}\right)<\infty
$$

The main technical result involved in the proof of Theorem 4 is the following version of the standard stationary phase estimates.

Lemma 5. Let $n \geq 3$. Let

$$
F_{j}(\xi)=\int_{\{y: 1 \leq|y| \leq 2\}} e^{i\left(\left\langle y, \xi^{\prime}\right\rangle+\xi_{n} \gamma_{j}(|y|)\right)} e^{i \xi_{n} / \gamma\left(2^{-j}\right)} d y,
$$

with $\gamma_{j}(s)=\gamma\left(2^{-j} s\right) / \gamma\left(2^{-j}\right)$, where $\gamma$ is as in (2). Then

$$
\left|F_{j}(\xi)\right| \leq C(1+|\xi|)^{-1},
$$

where $C$ is independent of $j$ and $\gamma$.

Moreover, if $\left|F_{j}(\xi)\right|$ is replaced by $\left|\nabla F_{j}(\xi)\right|$ then the estimate (13) still holds with $C$ on the right-hand side replaced by $C / \gamma\left(2^{-j}\right)$.

The main technical result used in the proof of Theorem 2 is the following. See [Bak95, Theorem 2.1].

Lemma 6. Let $\chi \in C_{0}^{1}([0, \infty))$ be a non-negative function that is compactly supported in the interval $(a, \infty)$, where $a>0$. Let $n=3$ and let $S$ be as in (2) where $\gamma$ satisfies the condition of Theorem 2. Let 
$F_{S}(\chi)(\xi)$ denote $F_{0}(\xi)$ in Lemma 5 with $\chi(|y|)$ in place of the characteristic function of the annulus $\{y: 1 \leq|y| \leq 2\}$.

Then for every multi-index $\alpha$ with $|\alpha| \leq 1$ there exists a constant $C$ independent of $a, \xi$, and $\chi$ such that

$$
\left|\left(\frac{\partial}{\partial \xi}\right)^{\alpha} F_{S}(\chi)(\xi)\right| \leq C C_{\chi} \frac{a}{\sqrt{\gamma^{\prime}(a) \gamma^{\prime}\left(\frac{a}{2}\right)}}(1+|\xi|)^{-1}
$$

where $C_{\chi} \leq\|\chi\|_{\infty}+\left\|\chi^{\prime}\right\|_{1}$ if $\alpha=0$, and $C_{\chi} \leq\|\chi\|_{\infty}+\|\chi\|_{1}+\left\|\chi^{\prime}\right\|_{1}$ if $\alpha=1$.

\section{Main idea.}

The point is that even though a higher dimensional analog of Lemma 6 may be difficult to obtain, we get around the problem by using Lemma 5. We have to settle for the uniform decay of order $\max \{-(n-2) / 2,-1\}$ instead of $-(n-1) / 2$, but this is enough in dimension $n \geq 4$ as we shall see below. The idea is, roughly speaking, the following. We are trying to prove $L^{\Phi} \longrightarrow L^{\Phi}$ estimates for maximal operators associated to radial convex surfaces. If the surface is infinitely flat, then [IoSa96, Theorem 2] implies that $L^{p} \longrightarrow L^{p}$ estimates are not possible for $p<\infty$. So we are looking for $L^{\Phi} \longrightarrow L^{\Phi}$ estimates where $L^{\Phi}$ is very close to $L^{\infty}$, so interpolating between $L^{2}$ and $L^{\infty}$ in the right way should do the trick. However, in order to obtain $L^{2}$ boundedness of the maximal operator, we only need decay $-1 / 2-\varepsilon, \varepsilon>0$. If $n \geq 4$, then $(n-2) / 2>1 / 2$, so we should be alright. If $n=3$ a bit more integration by parts will be required.

\section{Plan.}

The rest of the paper is organized as follows. In the next section we shall prove Theorem 4 assuming Lemma 5 . In the following section we shall prove Lemma 5 . In the final section of the paper we shall discuss the sharpness of Theorem 4 and give some examples. 


\section{Proof of Theorem 4.}

Let

$$
A_{t}^{j} f(x)=\int f\left(x^{\prime}-t y, x_{n}-t(\gamma(|y|)+1)\right) \psi_{0}(y) d y
$$

where $\psi_{0}$ is a smooth cutoff function supported in $[1,2]$, such that $\sum_{j} \psi\left(2^{j} s\right) \equiv 1$. Let $\tau_{j} f(x)=f\left(2^{-j} x^{\prime}, \gamma\left(2^{-j}\right) x_{n}\right)$. Making a change of variables we see that

$$
A_{t}^{j} f(x)=2^{-j(n-1)} \tau_{j}^{-1} B_{t}^{j} \tau_{j} f(x),
$$

where

$$
B_{t}^{j} f(x)=\int f\left(x^{\prime}-t y, x_{n}-t\left(\frac{\gamma_{j}(|y|)+1}{\gamma\left(2^{-j}\right)}\right)\right) \psi_{0}(y) d y
$$

We shall prove that

$$
\sup _{t>0} B_{t}^{j}: L^{2}\left(\mathbb{R}^{n}\right) \longrightarrow L^{2}\left(\mathbb{R}^{n}\right) \quad \text { with norm }\left(\frac{1}{\gamma\left(2^{-j}\right)}\right)^{1 / 2}
$$

By interpolating with the trivial estimate $\left\|\sup _{t>0} B_{t}^{j} f\right\|_{\infty} \leq C\|f\|_{\infty}$ using Lemma 3, we shall conclude that

$$
\sup _{t>0} B_{t}^{j}: L^{\Phi}\left(\mathbb{R}^{n}\right) \longrightarrow L^{\Phi}\left(\mathbb{R}^{n}\right) \quad \text { with norm } \Phi^{-1}\left(\frac{1}{\gamma\left(2^{-j}\right)}\right)
$$

Since the $L^{p}$ norms of $\tau_{j}$ and $\tau_{j}^{-1}$ are reciprocals of each other, it follows that $\mathcal{A}: L^{\Phi}\left(\mathbb{R}^{n}\right) \longrightarrow L^{\Phi}\left(\mathbb{R}^{n}\right)$ if

$$
\sum_{j=0}^{\infty} 2^{-j(n-1)} \Phi^{-1}\left(\frac{1}{\gamma\left(2^{-j}\right)}\right)<\infty .
$$

So it remains to prove (18). The proof follows from the standard Sobolev imbedding theorem type argument. See for example [St76]. We shall use the following version which follows from the proof of [IoSa96, Theorem 15]. See also, for example, [CoMa86], [MaRi95]. 
Lemma 7. Suppose that $\tau$ is the Lebesgue measure on the hypersurface $S$ supported in an ellipsoid with eccentricities $(1, \ldots, 1, R)$. Suppose that $|\hat{\tau}(\xi)| \leq C$ and $\max \{|x|: x \in \operatorname{supp}(\tau)\} \leq 10 R$. Suppose that

$$
\left(\int_{1}^{2}|\hat{\tau}(t \xi)|^{2} d t\right)^{1 / 2} \leq C(1+|\xi|)^{-1 / 2-\varepsilon}
$$

and

$$
\left(\int_{1}^{2}|\nabla \hat{\tau}(t \xi)|^{2} d t\right)^{1 / 2} \leq C R(1+|\xi|)^{-1 / 2-\varepsilon}
$$

for some $\varepsilon>0$. Let $\hat{\tau}_{t}(\xi)=\hat{\tau}(t \xi)$. Let $\mathcal{M} f(x)=\sup _{t>0}\left|f * \tau_{t}(x)\right|$. Then

$$
\|\mathcal{M} f\|_{2} \leq 100 C \sqrt{R}\|f\|_{2} .
$$

Application of Lemma 7 immediately yields (17) since by Lemma 5 $C$ is a universal constant and $R \leq C / \gamma\left(2^{-j}\right)$. This completes the proof of Theorem 4.

\section{Proof of Lemma 5 .}

We must show that

$$
\begin{aligned}
\left|F_{j}(\xi)\right| & =\left|\int_{\{y: 1 \leq|y| \leq 2\}} e^{i\left(\left\langle y, \xi^{\prime}\right\rangle+\xi_{n} \gamma_{j}(|y|)\right)} e^{i \xi_{n} / \gamma\left(2^{-j}\right)} d y\right| \\
& \leq C|\xi|^{-1}
\end{aligned}
$$

with $C$ independent of $\gamma$ and $j$.

Our plan is as follows. We will first show that if either $\left|\xi^{\prime}\right| \approx\left|\xi_{n}\right|$, or $\left|\xi^{\prime}\right| \gg\left|\xi_{n}\right|$, then $\left|F_{j}(\xi)\right| \leq C(1+|\xi|)^{-(n-2) / 2}$. If $\left|\xi_{n}\right| \gg\left|\xi^{\prime}\right|$, we will show that $\left|F_{j}(\xi)\right| \leq C\left(1+\left|\xi_{n}\right|\right)^{-1}$. This will complete the proof since $(n-2) / 2 \geq 1$ if $n \geq 4$.

Going into polar coordinates and applying stationary phase, we get

$$
e^{i \xi_{n} / \gamma\left(2^{-j}\right)} \int_{1}^{2} e^{i \xi_{n} \gamma_{j}(r)} r^{n-2} d r \int_{S^{n-2}} e^{i r\left\langle\xi^{\prime}, \omega\right\rangle} d \omega .
$$


Since the Gaussian curvature on $S^{n-2}$ does not vanish, it is a classical result that

$$
\left|\int_{S^{n-2}} e^{i\left\langle\xi^{\prime}, \omega\right\rangle} d \omega\right| \leq C\left(1+\left|\xi^{\prime}\right|\right)^{-(n-2) / 2}
$$

It follows that $\left|F_{j}(\xi)\right| \leq C(1+|\xi|)^{-n-2 / 2}$ if either $\left|\xi^{\prime}\right| \gg\left|\xi_{n}\right|$ or $\left|\xi^{\prime}\right| \approx$ $\left|\xi_{n}\right|$. If $\left|\xi_{n}\right| \gg\left|\xi^{\prime}\right|$, let $h(r)=\xi_{n} \gamma_{j}(r)-r\left\langle\xi^{\prime}, \omega\right\rangle$. Since $\gamma$ is convex, it follows that $\left|h^{\prime}(r)\right| \geq\left|\xi_{n}\right|-\left|\xi^{\prime}\right|$. Since $\left|\xi_{n}\right| \gg\left|\xi^{\prime}\right|$, it follows by the van der Corput Lemma that the expression in (24) is bounded by $C /|\xi|$.

The estimate for $\nabla F_{j}$ follows in the same way if we observe that the derivative with respect to $\xi_{n}$ brings down a factor of $\gamma_{j}(r)+1 / \gamma\left(2^{-j}\right)$, and $\gamma_{j}(r)+1 / \gamma\left(2^{-j}\right) \leq 2 / \gamma\left(2^{-j}\right)$. This completes the proof of Lemma 5 if $n \geq 4$.

To prove the three dimensional case we go into polar coordinates, integrate in the angular variables and use the well known asymptotics for the Fourier transform of the Lebesgue measure on the circle to obtain

$$
\int e^{i \phi(r)} r b(r A) \psi_{0}(r) d r
$$

where $A=\left|\xi^{\prime}\right|, \lambda=\xi_{n}, b$ is a symbol of order $-1 / 2, \psi_{0}$ is as above, and $\phi(r)=r A-\gamma_{j}(r) \lambda$.

Let

$$
G(r)=\int_{r}^{2} e^{i \phi(s)} d s
$$

so the integral in (26) becomes

$$
\int G^{\prime}(r) r b(r A) \psi_{0}(r) d r
$$

Integrating by parts we get

$$
\int G(r)\left(r b(r A) \psi_{0}(r)\right)^{\prime} d r
$$

Let $r_{0}$ be defined by the relation $\gamma_{j}^{\prime}\left(r_{0}\right)=A /(2 \lambda)$. We have $\left|\phi^{\prime \prime}(s)\right| \geq$ $\left|\gamma_{j}^{\prime \prime}(s) \lambda\right| \geq\left|\gamma_{j}^{\prime}(s) \lambda\right| \geq\left|\gamma_{j}^{\prime}(r) \lambda\right|$. If $r_{0}<r$ this quantity is bounded below by $C|A|$ and the van der Corput lemma gives the decay $C|A|^{-1 / 2}$ for $G(r)$. Using the fact $b$ is a symbol of order $-1 / 2$ we see that $(29)$ is 
bounded by $C|A|^{-1},|A|$ large. This handles the case $|\lambda| \leq C|A|$ and $r \leq r_{0}$.

On the other hand, $\left|\phi^{\prime}(s)\right|=\left|A-\gamma_{j}^{\prime}(s) \lambda\right|$. Split up the integral that defines $G(r)$ into two pieces: $s \in\left[r, r_{0}\right]$ and $s \in\left[r_{0}, 2\right]$. The second integral was just handled above. In the first integral $\left|\phi^{\prime}(s)\right| \geq\left|\phi^{\prime}\left(r_{0}\right)\right| \geq$ $C|A|$. The van der Corput lemma yields decay $C /|A|$. Taking the properties of the symbol $b$ into account, as before, we get the decay $C|A|^{-1 / 2} /|A|$. This takes care of the case $|\lambda| \leq C|A|$ and $r \geq r_{0}$.

If $|\lambda| \gg|A|,\left|\phi^{\prime}(s)\right| \geq C|\lambda|$ and the van der Corput lemma yields the decay $C /|\lambda|$ for (29). This completes the proof of the three dimensional case.

\section{Examples.}

EXAmple 1. Let $\gamma(s)=s^{m}, m \geq 2(n-1)$, and $\Phi(t)=t^{p}$. Theorem 4 yields boundedness for $p>m /(n-1)$. This is sharp by Theorem 1 .

EXAMPLE 2. Let $\gamma(s)=s^{m}, m \geq 2(n-1)$, and $\Phi_{p, \alpha}(s)=s^{p} \log ^{\alpha}(s)$. Then Theorem 4 yields boundedness for $p=m /(n-1)$ and $\alpha>m /(n-1)$.

ExAmple 3. Let $\gamma(s)=e^{-1 / s^{\alpha}}, \alpha>0$, and $\Phi(t)=e^{t^{\beta}}, \beta>0$. Then Theorem 4 tells us that the maximal operator is bounded if $\alpha<$ $\beta(n-1)$. Testing $A_{t} f(x)$ against

$$
h_{p}(x)=\Phi^{-1}\left(\frac{1}{\left|x_{n}\right|}\right) \frac{1}{\log \left(\frac{1}{\left|x_{n}\right|}\right)} \chi_{B}(x),
$$

where $\chi_{B}$ is the characteristic function of the ball of radius $1 / 2$ centered at the origin, shows that this result is sharp. The same procedure establishes sharpness of the estimate given in Example 2.

In fact, testing $A_{t} f(x)$ against $h_{p}(x)$ shows that the summation condition of Theorem 4 is pretty close to being sharp. It is not hard to see that, at least up to a log factor, $\mathcal{A}$ bounded on $L^{\Phi}\left(\mathbb{R}^{n}\right)$ implies that

$$
\int_{\{y:|y| \leq 2\}} \Phi^{-1}\left(\frac{1}{\gamma(|y|)}\right) d y<\infty .
$$

This would literally follow, without the log factor, from the proof of [IoSa96, Theorem 2] if we assumed, in addition, that $\Phi(a b) \geq \Phi(a) \Phi(b)$, for every $a, b>0$. 
The condition (30) is equivalent (after making a change of variables and going into polar coordinates) to

$$
\sum_{j=0}^{\infty} 2^{-j(n-1)} \int_{1}^{2} \Phi^{-1}\left(\frac{1}{\gamma\left(2^{-j} r\right)}\right) r^{n-2} d r<\infty .
$$

The expression (31) is equivalent to the summation condition of Theorem 4 if $\gamma$ does not vanish to infinite order. If $\gamma$ vanishes to infinite order, the two conditions are still often equivalent, as in the Example 3 above.

REMARK. It would be interesting to extend the results of this paper to a more general class of hypersurfaces. For example, one could consider hypersurfaces of the form $S=\left\{x \in \mathbb{R}^{n}: x_{n}=\gamma\left(\phi\left(x^{\prime}\right)\right)+1\right\}$ where $\gamma$ is as above and $\phi$ is a smooth convex finite type function. Some recent results (see e.g. [IoSa97], [IoSaSe98], and [WWZ97]) suggest that such an analysis should be possible. We shall address this issue in a subsequent paper ([I98]). More generally, a bigger challange would be to consider a hypersurface of the form $S=\left\{x \in \mathbb{R}^{n}: x_{n}=G\left(x^{\prime}\right)+1\right\}$, where $G$ is a smooth function of $n-1$ variables that vanishes of infinite order at the origin. At the moment, obtaining sharp Orlicz estimates, even in the case where the determinant of the Hessian matrix of $G$ only vanishes at the origin, does not seem accessible.

Acknowledgements. The author wishes to thank Jim Wright for teaching him the technique needed to prove the three dimensional case of Lemma 5 above.

\section{References.}

[Bak95] Bak, J.-G., Averages over surfaces with infinitely flat points. J. Funct. Anal. 129 (1995), 455-470.

[B86] Bourgain, J., Averages in the plane over convex curves and maximal operators. J. Analyse Math. 47 (1986), 69-85.

[CoMa86] Cowling, M., Mauceri, G., Inequalities for some maximal functions II. Trans. Amer. Math. Soc. 296 (1986), 341-365.

[Gr82] Greenleaf, A., Principal curvature and harmonic analysis. Indiana Math. J. 30 (1982), 519-537. 
[I94] Iosevich, A., Maximal operators associated to families of flat curves in the plane. Duke Math. J. 76 (1994), 633-644.

[198] Iosevich, A., Averages over convex infinite type hypersurfaces. Preprint.

[IoSa96] Iosevich, A., Sawyer, E., Oscillatory integrals and maximal averaging operators associated to homogeneous hypersurfaces. Duke Math. J. 82 (1996), 103-141.

[IoSa97] Iosevich, A., Sawyer, E., Maximal averages over surfaces. Advances in Math. 132 (1997), 46-119.

[IoSaSe98] Iosevich, A., Sawyer, E., Seeger, A., Averages over convex surfaces of finite type in $\mathbb{R}^{3}$. Preprint, 1998 .

[MaRi95] Marletta, G., Ricci, F., Two parameter maximal functions associated with homogeneous surfaces in $\mathbb{R}^{n}$. Studia Math. 130 (1998), 53-65.

[St76] Stein, E. M., Maximal functions: spherical means. Proc. Nat. Acad. Sci. USA $\mathbf{7 3}$ (1976), 2174-2175.

[St93] Stein, E. M., Harmonic Analysis. Princeton Univ. Press, 1993.

[WWZ97] Wright, J., Wainger, S., Ziesler, S., Preprint.

Recibido: 29 de octubre de 1.997

\author{
Alex Iosevich* \\ Department of Mathematics \\ Georgetown University \\ Washington D.C. 20057 \\ iosevich@math.georgetown.edu
}

\footnotetext{
* Research at MSRI supported in part by the NSF grant DMS97-06825
} 